\title{
Plan de formación para el manejo de entornos virtuales para los docentes de la Universidad de Guayaquil
}

\section{Training plan to handle virtual environments for teachers of the University of Guayaquil}

Jorge Misael Merchán Riera

Universidad de Guayaquil, Ecuador

Johanna Patricia Zumba Gamboa

Universidad de Guayaquil, Ecuador

Autor para correspondencia: jorge.merchanr@ug.edu.ec, johanna.zumbag@ug.edu.ec Fecha de recepción: 3 de abril del 2018 - Fecha de aceptación: 15 de agosto del 2018

\section{Resumen}

Este artículo tiene como objetivo presentar una propuesta de formación para los profesores universitarios que les permita manejar entornos virtuales como apoyo a los procesos de enseñanza y aprendizaje, en la Universidad de Guayaquil en Ecuador; en específico en la carrera Ingeniería en Sistemas Administrativos Computarizado de la Facultad de Ciencias Administrativas. Se realizó una investigación descriptiva, de campo y transaccional, para diseñar el plan de formación que se aspira aplicar en el año 2018. Se concluye que es necesario que este plan de formación sea parte esencial del plan de carrera de los profesores de la Universidad de Guayaquil, para contribuir con la formación que requiere este nuevo milenio.

Palabras Claves: formación; entornos virtuales; procesos de enseñanza y aprendizaje; Universidad de Guayaquil

\begin{abstract}
This article aims to present a proposal for training for university teachers to enable them to handle virtual environments to support the processes of teaching and learning at the University of Guayaquil in Ecuador; specifically in the career in Computerized Management Systems Engineering at the Faculty of Administrative Sciences. A descriptive research, field study and transectional was held to design the training plan that aims to apply in 2018. It is concluded that it is necessary that this training is an essential part of the career plan for teachers at the University of Guayaquil, to contribute to the training required by this new millennium.
\end{abstract}

Key words: training; virtual environments; teaching and learning; University of Guayaquil 


\section{Introducción}

La nueva sociedad del conocimiento requiere de profesionales formados para cubrir con las expectativas que esta plantea. Por ello, es necesario que las personas no solo estén preparadas para generar conocimientos, sino también para manejar las tecnologías de información y comunicación (TIC) y de esa manera integrarse a un mundo conectado, hiperinformado, con alta carga tecnológica y con necesidades de comunicación que cada vez varían según el mundo se va transformando.

Esta sociedad, ha exigido cambios sociales, políticos, económicos y educativos, que representan retos importantes para los inmigrantes digitales, ya que estos debido a su edad no han vivido intensamente la avalancha tecnológica, pero se han visto en la necesidad de usar las TIC y mantenerse al día para competir en un mundo cada vez más especializado. Esto los ha llevado a formarse en el mundo tecnológico, a adaptarse a los cambios pero siempre manteniendo conexión con el pasado, ellos tienen la comprensión de los dos mundos (antesdespués de Internet) (Prensky, 2001).

La mayoría de los profesores que dictan clases en la carrera de Ingeniería en Sistemas Administrativos Computarizados en la Universidad de Guayaquil son inmigrantes digitales; por ello, están altamente formados para la educación presencial y poseen escasa formación en el uso de las nuevas tecnologías. Sin embargo, ellos consideran que en un inicio se puede comenzar con incorporar el uso de Moodle, en la modalidad de apoyo a la presencialidad, lo cual permitiría solucionar algunos inconvenientes que se dan en la actualidad como lo son, según Zumba y Merchán (2015):

a) Los tiempos reducidos en las horas de clases para explicar las asignaciones de trabajos autónomos a los estudiantes, ya que es necesario completar el contenido del programa de estudio en las horas de clases por lo extenso del mismo;

b) La falta de soporte al estudiante entre clases para ayudarlo a comprender mejor los contenidos, ya que no se cuentan con los mecanismos e infraestructuras necesarias para ello.

c) La poca interacción permanente entre los estudiantes fueras de las horas de clase, que les permita aprender colaborativamente.

Por ello, es necesario generar un plan de formación que cubra no solo con lo requerido por la universidad, sino que este diseñado para este tipo de personas, los inmigrantes digitales; los cuales tienen una perspectiva particular de las TIC y requieren comprender muchos elementos asociados a estas y su aplicación en el mundo educativo, así como, comenzar por elementos básicos asociados al uso de las TIC.

Este estudio forma parte del proyecto que pretende impulsar la incorporación de esta carrera a la modalidad educación a distancia y virtual. En la primera parte de este proyecto, se determinó la disposición que tenían los profesores a dictar la asignatura en un ambiente virtual, para ello se entrevistó a trece profesores de una plantilla de treinta y dos obteniéndose como resultados que requiere pasar por procesos de promoción y educación interna de los profesores 
sobre lo relativo, no solo a Moodle sino a los conceptos básicos asociados al uso de las TIC como apoyo a los procesos de enseñanza y aprendizaje como un paso inicial y que estos tienen una alta disposición hacia la preparación y a abordar un proyecto de este tipo.

Cabe destacar, que aunque la Universidad de Guayaquil posee carreras que se dictan a distancia y virtual, y que existe un plan de formación para los profesores que trabajan en esta modalidad, no se ha asumido el uso de Moodle como apoyo a la presencialidad y este plan de formación pretende abordar este aspecto, para solventar problemas como: a) la falta de tiempo que tienen los estudiantes que trabajan para dedicarse a sus estudios, b) permitir que personas que tienen horarios complicados de trabajo -turnos rotativos, horarios especiales, entre otros- o madres solteras que trabajan y desean estudiar puedan tener acceso a la educación universitaria y c) dificultad de acceso a la educación a las personas que viven o trabajan alejadas de la comunidad (Zumba y Merchán, 2015).

Así pues, con esta propuesta se pretende dar solución a los problemas antes mencionados, así como lograr que a mediano plazo la carrera pueda ser impartida en la modalidad a distancia y virtual y que los profesores que ya dictan las asignaturas en la presencialidad puedan ser considerados para la modalidad virtual.

La estrategia metodológica utilizada para desarrollar esta capacitación es la propuesta por Pineda (2013), la cual está conformada por cinco fases que permiten mejorar el proceso educativo y está estructurada en de la siguiente manera:

El análisis permite detectar necesidades, definir las características, objetivos y el perfil de los participantes... diseño de las estrategias, la metodología y el esquema base que soportan la formación... en la etapa de desarrollo: se plasma un concepto en función de las necesidades encontradas y las estrategias y los objetivos planteados... y su posterior implementación en dos fases, ya que se prevé finalizar con un ciclo de evaluación de los resultados por los docentes capacitados (pp.115-116).

Pineda (2013) plantea una implementación en dos etapas, ya que la formación debe ir midiendo los avances paulatinos de los profesores, para ir profundizando en los aspectos de formación que requieren un cambio de paradigma en el proceso de enseñanza y aprendizaje para la virtualidad. Además plantea, que son necesarios avances en el hacer de los profesores con la herramienta, para comprender otros aspectos más complejos como la moderación en línea y el tema de la evaluación.

\section{Desarrollo}

\section{Las bases del plan de formación}

En este apartado se presentan los resultados de la primera fase de la metodología: el Análisis, la cual permite la comprensión del perfil de las personas que tomaran la capacitación para ir adaptando la formación al nivel del grupo. 
El primer elemento que se consideró en esta propuesta, está ligado al perfil de los docentes; en este caso inmigrantes digitales, pues en promedio las edades de los profesores que dictan las asignaturas están alrededor de los 35 años, por ello:

es de vital importancia tomar en consideración que los inmigrantes digitales deben utilizar en menor medida las metodologías centradas en el profesor (caracterizadas como expositivas y pasivas) para ir evolucionando hacia metodologías centradas en el estudiante (activas, dinámicas y participativas) (Pineda, 2013:110).

En este sentido, es necesario sensibilizar al docente no solo en el manejo de herramientas informáticas, sino también en los elementos básicos de la educación a distancia. Ese paradigma que permite comprender que el docente deja de ser el poseedor del conocimiento, para ser un facilitador en el proceso de enseñanza y aprendizaje.

Esto se debe, a que los docentes han tenido poco contacto con la educación a distancia, tanto en el rol de profesor o como de alumno, ya que sólo un 10\% de los participantes ha tomado cursos a distancia y el $20 \%$ de ellos han impartido clases usando Moodle como plataforma de aprendizaje.

Para definir que herramientas informáticas dominaban los docentes, se realizó una encuesta, arrojando los siguientes resultados:

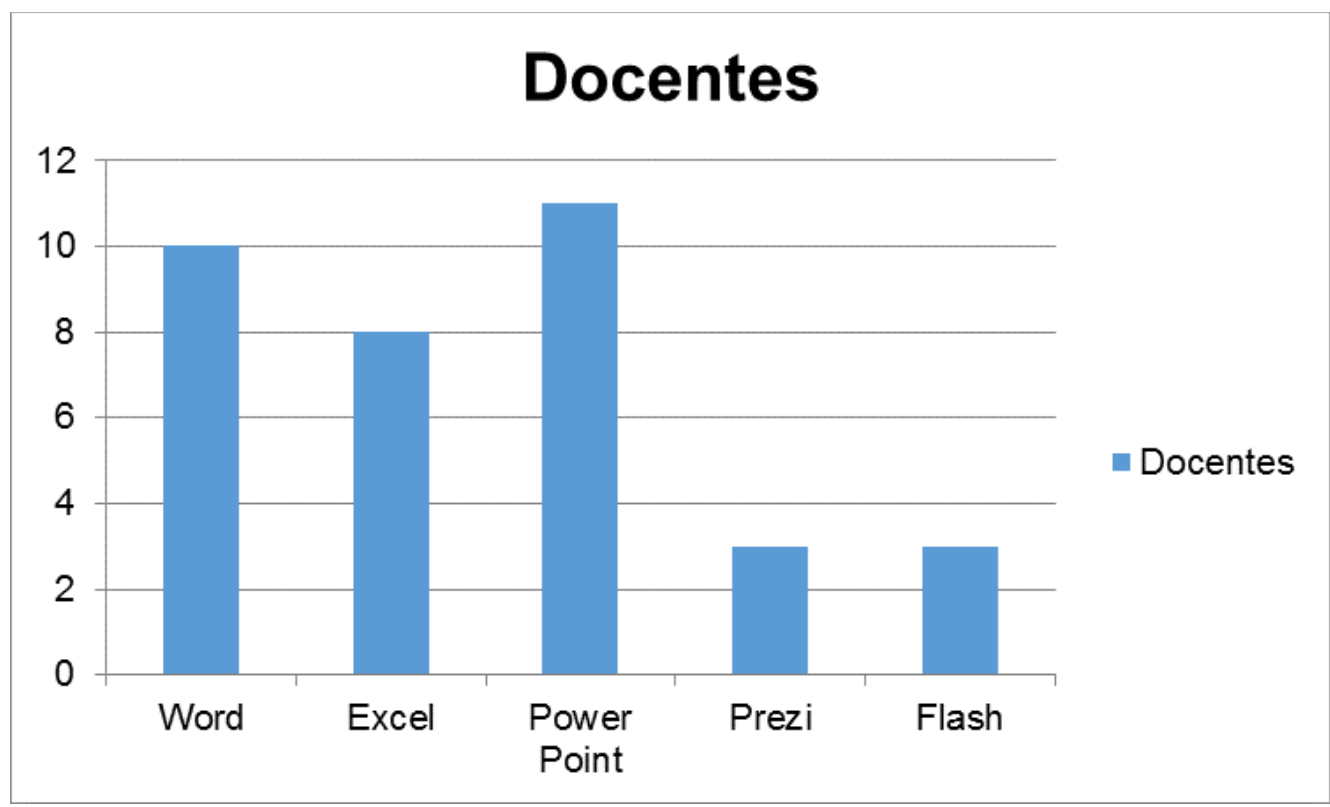

Figura No. 1. Herramientas informáticas dominadas por los docentes Fuente propia.

Se puede observar que las herramientas que más dominan los profesores son Word y Power Point, esto tuvo una ventaja significativa en el diseño de la propuesta, ya que para estas 
herramientas se programaron cursos avanzados que permitieran a los profesores estructurar guías didácticas y presentaciones apropiadas para la educación a distancia.

En el caso de Excel y Flash, se consideró realizar cursos básico, medio y avanzado, que permitieran al docente crear herramientas de trabajo en las asignaturas, como hojas de cálculos para resolver problemas de estadística, cálculo, matemáticas, entre otras asignaturas; y crear animaciones para comenzar a construir una base de datos de objetos de aprendizajes tipo flash, para asignaturas como redes, arquitectura computacional, programación, entre otras.

Con respecto al manejo del Moodle, el $77 \%$ de los profesores conoce la herramienta pero solo el $46 \%$ ha recibido algún tipo de capacitación para el manejo de esta. Denotando esto que, en la capacitación es necesario comenzar desde lo más básico en el proceso de enseñanza sobre Moodle. Con respecto al conocimiento sobre el manejo de los recursos ofrecidos por Moodle para la construcción de las sesiones académicas es escaso, solo se conoce cómo añadir una etiqueta, editar una página de texto, editar una página web o montar archivos como recursos.

Así pues, se conceptualizaron varias actividades que le permitirán al profesor no solo aprender a usar la herramienta, sino como aplicarla para el proceso de enseñanza y aprendizaje.

Por ello, para el desarrollo del programa se consideró que tuviese en un esquema de 50\% de presencialidad y $50 \%$ de virtualidad, con esto se logrará que los profesores no solo se hagan consciente de su papel como facilitador, sino también como estudiante, para que puedan comprender el proceso de la educación virtual desde ambas perspectivas.

Otro elemento importante para el diseño del plan de formación, fue que el 93\% de los profesores estuvieron de acuerdo con tomar capacitaciones para emprender este proceso de usar las TIC como apoyo a la docencia y luego ir hacia la educación virtual.

Cabe destacar, que el $38 \%$ de los profesores consideran que la mejor modalidad es $60 \%$ presencial y $40 \%$ virtual y un $31 \%$ considera $80 \%$ presencial y $20 \%$ virtual, el resto piensa que $50 \%$ es la opción más viable; por lo que se puede observar que hay una actitud mayor a apoyar más la presencialidad que la virtualidad.

Este elemento, llamo a la reflexión a los autores y generó la incorporación de una sección de sensibilización de los profesores hacia la educación virtual, a fin de que estos puedan pensar en un futuro en la posibilidad de que su materia se dicte totalmente en la virtualidad.

Cuando se evaluó sobre las responsabilidades de administración del aula virtual de la asignatura, el $85 \%$ de los profesores indican que la administración debe recaer primeramente en el coordinador de la misma, sin embargo el $70 \%$ cree que cualquier profesor de la asignatura podría ser el administrador.

Esto permite observar que los encuestados están abiertos a que cualquier profesor de la asignatura asuma esta responsabilidad; sin embargo, este hecho parece indicar que los profesores no tienen claro lo que esto realmente significa; por lo que se estableció en el plan de formación un espacio de reflexión sobre las responsabilidades de los administradores de las aulas virtuales y 
de los profesores de la asignatura para que la educación a distancia pueda funcionar de manera apropiada.

El tercer elemento importante para esta formación, es la conformación de los grupos de profesores que estarían interesados en la formación, los cuales se segmentaran de acuerdo a su nivel de conocimiento o experticia en el área de las TIC,

ya que el uso de las tecnologías Web 2.0 entre nativos e inmigrantes digitales en el ámbito socioeducativo puede ayudar y potenciar la constitución y consolidación de equipos de trabajo, facilitar el diálogo e intercambio de ideas, experiencias entre los diferentes grupos sociales, la indagación e investigación educativa, la reflexión colectiva, la interacción y la participación social (Pineda, 2013:115).

\section{El diseño: la arquitectura del plan de formación}

Para este plan de formación, tomando como referencia las fases propuestas por Pineda (2013), se establecieron 5 fases las cuales se llevarán de manera secuencial, con evaluación y retroalimentación por parte de los participantes.

Fase I. Sensibilización: es necesario que el docente entienda y comprenda lo que significa la educación a distancia, no solo en beneficios y oportunidades, sino el cambio que esto implica en el proceso de enseñanza y aprendizaje.

Para ello, se consideró que una de las mejores estrategias para la sensibilización, es el uso de la multimedia y los videos, en conjunto con los foros como estrategia de socialización, su duración es de 16 horas académicas. Lo interesante de este proceso, es que algunos foros serán presenciales y otros a través de la virtualidad. Para ello, se usará Moodle como plataforma de educación a distancia, con el objetivo que los profesores se integren a la modalidad a distancia y al uso de los foros como herramienta para la discusión en clase.

A continuación se muestra la siguiente tabla, en donde se puede apreciar las actividades a desarrollar, su modalidad, duración, recursos empleados y estrategia utilizada.

Tabla 1. Actividades de la fase de sensibilización

\begin{tabular}{|c|c|c|c|c|}
\hline No. & Actividad/Estrategia & $\begin{array}{c}\text { Tipo } \\
\text { actividad }\end{array}$ & $\begin{array}{c}\text { Recurso } \\
\text { disponible en }\end{array}$ & $\begin{array}{c}\text { No. } \\
\text { de } \\
\text { horas }\end{array}$ \\
\hline 1. & $\begin{array}{l}\text { Foro: Ventajas de la Educación a Distancia. } \\
\text { El participante debe ver, previamente a la asistencia al foro, los } \\
\text { recursos habilitados en el Aula virtual, en la fase de } \\
\text { Sensibilización, etiqueta Primera actividad: Ventajas de la } \\
\text { Educación a Distancia. }\end{array}$ & Mixta & $\begin{array}{l}\text { Videos en } \\
\text { Moodle. } \\
\text { Foro } \\
\text { Presencial. }\end{array}$ & 4 \\
\hline
\end{tabular}


2. Foro: La educación del mañana y los cambios tecnológicos. El participante deber ver las películas asignadas y participar en las discusiones abiertas en la plataforma, a través de los foros. Los recursos están habilitados en el Aula virtual, en la fase de Sensibilización, etiqueta Segunda actividad: La Educación del mañana y los cambios tecnológicos.

3. Foro: El cambio paradigmático en la educación.

El participante debe ver, previamente a la asistencia al foro, los recursos habilitados en el Aula virtual, en la fase de Sensibilización, etiqueta Tercera actividad: El cambio paradigmático en la educación.

$4 \quad$ Llenado del formato de evaluación de la fase.

El participante llenará una encuesta programada en la plataforma, para evaluar tanto las actividades como su participación y la de sus compañeros de actividades. El recurso está disponible en la fase de Sensibilización, etiqueta Cuarta actividad: Evaluación.
Virtual
Videos y
foros en
Moodle.

$\begin{array}{clc}\text { Mixta } & \begin{array}{l}\text { Videos en } \\ \text { Moodle. }\end{array} & 4 \\ & \begin{array}{l}\text { Foro } \\ \text { Presencial. }\end{array} & \\ \text { Virtual } & \begin{array}{l}\text { Cuestionario } \\ \text { en Moodle. }\end{array} & 1\end{array}$

Fuente: elaboración propia.

Fase II. Manejo de Moodle: el objetivo de esta fase es capacitar a los profesores en el manejo de Moodle como plataforma para la educación virtual. Dado que esta formación tiene sus bases en comenzar desde la modalidad semipresencial, se darán las capacitaciones de manera presencial usando la plataforma en los perfiles de estudiantes, administradores de la asignatura y profesores. Su duración es de 16 horas, sin embargo se puede considerar la extensión de su duración en función de las necesidades de los participantes.

Es importante destacar, que los cursos se harán en 2 fases: la primera donde se impartirán los conceptos básicos para el manejo de Moodle y la segunda, luego de terminar la Fase III, para que los profesores puedan cargar los recursos desarrollados a sus aulas virtuales.

Tabla 2. Actividades de la fase manejo de moodle

\begin{tabular}{|c|c|c|c|c|}
\hline No. & Actividad/Estrategia & $\begin{array}{c}\text { Tipo } \\
\text { actividad }\end{array}$ & $\begin{array}{c}\text { Recurso } \\
\text { disponible en }\end{array}$ & $\begin{array}{c}\text { No. } \\
\text { de } \\
\text { horas }\end{array}$ \\
\hline 1. & $\begin{array}{l}\text { Taller: Conceptos básicos de Moodle. } \\
\text { El participante debe ver, previamente a la asistencia al taller, } \\
\text { los recursos habilitados en el Aula virtual, en la fase de Manejo } \\
\text { de Moodle, etiqueta Primera actividad: Conceptos básicos de } \\
\text { Moodle. }\end{array}$ & Mixta & $\begin{array}{c}\text { Videos en } \\
\text { Moodle. } \\
\text { Foro en Moodle. } \\
\text { Taller } \\
\text { presencial. }\end{array}$ & 6 \\
\hline
\end{tabular}




\section{Taller: Consolidando el Aula Virtual.}

El participante deber ver los recursos asignados, previamente a la asistencia al taller, y participar en las discusiones abiertas en la plataforma a través del foro. Los recursos están habilitados en el Aula virtual, en la fase de Manejo de Moodle, etiqueta Segunda actividad: Consolidando el aula virtual.

3

Llenado del formato de evaluación de la fase.

El participante llenará una encuesta programada en la plataforma, para evaluar tanto las actividades como su participación y la de sus compañeros de actividades. El recurso está disponible en la fase de Manejo de Moodle, etiqueta Tercera actividad: Evaluación.

\author{
Virtual \\ Videos en \\ Moodle. \\ Foro en Moodle. \\ Talleres \\ presenciales con \\ mesa de trabajo.
}

Virtual Cuestionario en Moodle.

Fuente: elaboración propia

Fase III. Elaboración de Materiales Educativos: tiene como objetivo preparar a los docentes en la construcción de los objetos de aprendizajes (OA) requeridos para dictar la asignatura que imparte. Para ellos, se organizaran a los profesores que dicten la misma asignatura para compartir la elaboración de los OA y así aunar esfuerzos para crear mejores herramientas didácticas. Su duración es de 60 horas.

En esta fase se enseña sobre: a) el manejo de la tecnología para elaborar los objetos de aprendizaje y b) sobre las características que tienen los OA en la virtualidad. Para su ejecución se concibieron las siguientes actividades:

Tabla 3. Actividades de la fase de elaboración de materiales educativos

\begin{tabular}{|c|c|c|c|c|}
\hline No. & Actividad/Estrategia & $\begin{array}{c}\text { Tipo } \\
\text { actividad }\end{array}$ & $\begin{array}{c}\text { Recurso } \\
\text { disponible en }\end{array}$ & $\begin{array}{c}\text { No. } \\
\text { de } \\
\text { horas }\end{array}$ \\
\hline 1. & $\begin{array}{l}\text { Foro: Adaptación de materiales de acuerdo a la } \\
\text { normativa de la Universidad de Guayaquil. } \\
\text { El participante debe leer, previamente a la asistencia al } \\
\text { foro, los recursos habilitados en el Aula virtual, en la fase } \\
\text { de Elaboración de Materiales Educativos, etiqueta } \\
\text { Primera actividad: Adaptación de los materiales. }\end{array}$ & Mixta & $\begin{array}{l}\text { Lecturas en } \\
\text { Moodle. } \\
\text { Foro presencial. }\end{array}$ & 7 \\
\hline 2. & $\begin{array}{l}\text { Actualización en Microsoft Office } \\
\text { El participante debe ver los recursos habilitados en el } \\
\text { Aula virtual antes de las mesas de trabajo, en la fase de } \\
\text { Elaboración de Materiales Educativos, etiqueta Segunda } \\
\text { actividad: Actualización en MS Office. } \\
\text { Nota: habrá en la plataforma un calendario para indicar } \\
\text { las fechas y horas de las mesas de trabajo y un foro para } \\
\text { discutir dudas sobre el uso de las herramientas } \\
\text { tecnológicas. }\end{array}$ & Mixta & $\begin{array}{l}\text { Videos en Moodle. } \\
\text { Foro en Moodle. } \\
\text { Mesas de trabajo } \\
\text { presenciales. }\end{array}$ & 12 \\
\hline
\end{tabular}




\section{Taller para la elaboración de Materiales Educativos}

Los participantes tendrán sus espacios en la plataforma para compartir los recursos de aprendizaje que estén desarrollando, de manera que puedan compartir los profesores de una misma asignatura, sino que otros puedan observar los avances realizados. La idea es comprender, no solo cómo hacer el material educativo, sino también como trabajar los conceptos de participación y colaboración, propios de la educación a distancia.

Existe un foro para discutir sobre dudas y estrategias para la elaboración de los materiales.

Los recursos están habilitados en el Aula virtual, en la fase de Elaboración de Materiales Educativos, etiqueta Tercera actividad: Elaboración de los materiales.
Mixta

Trabajo
colaborativo por la

plataforma.

Foro en Moodle.

Mesas de trabajo.
Virtual Cuestionario $\quad 1$ en Moodle.

Fuente: elaboración propia.

Fase IV. Evaluación en línea: el objetivo de esta fase es discutir con los docentes las mejores estrategias de evaluación a aplicar en la virtualidad. Para comenzar con esta fase, los profesores deben haber culminado el montaje y armado de sus aulas virtuales y tenerlas en funcionamiento en la modalidad apoyo a la presencialidad.

Se busca que, una vez que el profesor está usando el aula para compartir recursos y ejecutando actividades en línea con los estudiantes, se proceda a incorporar procesos de evaluación en línea a cada asignatura, usando cuestionarios, envió de tareas y otras estrategias de evaluación. Al finalizar esta fase, el profesor debe haber creado los procesos de evaluación en línea en el aula virtual de la asignatura.

Tabla 4. Actividades de la fase de evaluación en línea

\begin{tabular}{llccc}
\hline No. & \multicolumn{1}{c}{ Actividad/Estrategia } & $\begin{array}{c}\text { Tipo } \\
\text { actividad }\end{array}$ & $\begin{array}{c}\text { Recurso } \\
\text { disponible en }\end{array}$ & $\begin{array}{c}\text { No. } \\
\text { de } \\
\text { horas }\end{array}$ \\
\hline 1. $\quad \begin{array}{l}\text { Foro: Estrategias de evaluación. } \\
\text { El participante debe leer y ver, previamente a la asistencia } \\
\text { al foro, los recursos habilitados en el Aula virtual, en la fase } \\
\text { de Evaluación en línea, etiqueta Primera actividad: } \\
\text { Estrategias de evaluación. }\end{array}$ & Mixta & $\begin{array}{c}\text { Lecturas en } \\
\text { Moodle. }\end{array}$ & 3 \\
\end{tabular}




\begin{tabular}{|c|c|c|c|c|}
\hline 2. & $\begin{array}{l}\text { Taller: La evaluación en Moodle. } \\
\text { El participante debe leer y ver, previamente a la asistencia } \\
\text { a los talleres presenciales, los recursos habilitados en el Aula } \\
\text { virtual, en la fase de Evaluación en línea, etiqueta Segunda } \\
\text { actividad: Evaluación en Moodle. }\end{array}$ & Mixta & $\begin{array}{c}\text { Videos en } \\
\text { Moodle. } \\
\text { Lecturas. } \\
\text { Talleres } \\
\text { presenciales. }\end{array}$ & 8 \\
\hline 3. & $\begin{array}{l}\text { Llenado del formato de evaluación de la fase. } \\
\text { El participante llenará una encuesta programada en la } \\
\text { plataforma, para evaluar tanto las actividades como su } \\
\text { participación y la de sus compañeros de actividades. El } \\
\text { recurso está disponible en la fase de Evaluación en línea, } \\
\text { etiqueta Tercera actividad: Evaluación. }\end{array}$ & Virtual & $\begin{array}{l}\text { Cuestionario } \\
\text { en Moodle. }\end{array}$ & 1 \\
\hline
\end{tabular}

Fuente: elaboración propia.

Fase V. Moderación básica: el objetivo de esta fase es formar al profesor para dar respuesta de manera efectiva a los requerimientos de comunicación e información del curso y los estudiantes; brindándole herramientas para ser asertivos, colaborativos e impulsadores de procesos de aprendizajes abiertos, donde el docente se vuelve un facilitador en el proceso de enseñanza y aprendizaje. Su duración es de 16 horas.

Se busca que, una vez que el profesor está usando el aula para compartir recursos, ejecutar actividades y hacer evaluaciones, pueda transformarlo en un espacio efectivo para realizar discusiones, construir conocimiento colaborativamente y guiar al estudiante, según sus propias necesidades de aprendizaje y comprensión del conocimiento, a lograr los objetivos del curso. A continuación se presentan las actividades de la quinta fase:

\section{Tabla 5. Actividades de la fase moderación básica}

\begin{tabular}{|c|c|c|c|c|}
\hline No. & Actividad/Estrategia & $\begin{array}{c}\text { Tipo } \\
\text { actividad }\end{array}$ & $\begin{array}{c}\text { Recurso disponible } \\
\text { en }\end{array}$ & $\begin{array}{c}\text { No. } \\
\text { de } \\
\text { horas }\end{array}$ \\
\hline 1. & $\begin{array}{l}\text { Foro: Qué es la moderación. } \\
\text { El participante debe leer y ver, previamente a la } \\
\text { asistencia al foro, los recursos habilitados en el Aula } \\
\text { virtual, en la fase de Moderación básica, etiqueta Primera } \\
\text { actividad: Qué es la moderación.. }\end{array}$ & Mixta & $\begin{array}{l}\text { Lectura en Moodle. } \\
\text { Videos en Moodle. } \\
\text { Foro presencial. }\end{array}$ & 3 \\
\hline 2. & $\begin{array}{l}\text { Taller: Moderando en línea. } \\
\text { El participante debe leer y ver, previamente a la } \\
\text { asistencia a los talleres presenciales, los recursos } \\
\text { habilitados en el Aula virtual, en la fase de Evaluación en } \\
\text { línea, etiqueta Segunda actividad: Moderando en línea. } \\
\text { Igualmente deberá entregar las asignaciones a través de } \\
\text { la plataforma, en el recurso denominado: Entrega de } \\
\text { asignaciones. }\end{array}$ & Mixta & $\begin{array}{l}\text { Lecturas en Moodle. } \\
\text { Ejemplos prácticos } \\
\text { de moderación en } \\
\text { Moodle. } \\
\text { Videos en Moodle. } \\
\text { Talleres presenciales }\end{array}$ & 12 \\
\hline
\end{tabular}




$\begin{array}{llc}\text { 3. Llenado del formato de evaluación de la fase. } & \text { Virtual } & \text { Cuestionario } \\ \text { El participante llenará una encuesta programada en la } & \text { endle. } \\ \text { plataforma, para evaluar tanto las actividades como su } & \\ \text { participación y la de sus compañeros de actividades. El } & \\ \text { recurso está disponible en la fase de Evaluación en línea, } & \\ \text { etiqueta Tercera actividad: Evaluación. } & \end{array}$

Fuente: elaboración propia.

\section{Conclusiones}

Es necesario que la Universidad de Guayaquil diseñe un plan de formación que se adapte a los inmigrantes digitales, con lo cual los profesores de la carrera Ingeniería en Sistemas Administrativos Computarizado de la Facultad de Ciencias Administrativas, puedan ir comprendiendo la educación a distancia e ir aplicándola de manera gradual como apoyo a la presencialidad.

Esto garantizaría el éxito del programa de formación, así como facilitaría este proceso en otras carreras que quieran realizar el mismo esfuerzo, algo que está cercano a ocurrir por la dinámica de la sociedad ecuatoriana actual, que obliga a la Universidad a mirar de frente las tendencias de la semipresencialidad como una estrategia de fortalecimiento del proceso educativo actual.

Es importante para este proceso la apertura que tienen los profesores a formarse en el área de las TIC, eso demuestra receptividad y disposición por parte de los profesores para comenzar en este proceso de incorporar las TIC como apoyo a la presencialidad.

El hecho de que el plan sea concebido para inmigrantes digitales, hará mucho más fácil el proceso de enseñanza aprendizaje y permitirá paulatinamente ir incorporando al profesor en los temas de la virtualidad en la educación y su aplicación.

En este sentido, la fase de Sensibilización es un elemento importante dentro del plan de formación, pues permitirá que el docente pueda comprender todo el proceso de enseñanza y aprendizaje en la virtualidad; y a través de las diferentes actividades diseñadas, se pretende que en esta capacitación el profesor experimente el modelo de semipresencialidad, para que se le haga más sencillo aplicarlo en su asignatura.

Aunado a esto, el diseño del plan de formación pretende que al terminar la formación el docente tenga terminado el montaje y armado de su aula virtual para el apoyo de la presencialidad, de manera que el profesor pueda empezar a usar su aula virtual al concluir.

Con el diseño innovador de este plan de formación, se espera que este sirva de guía y apoyo para seguir formando a los profesores de la facultad en materia de TIC y sea considerado para formar parte del plan de carrera que ofrece la universidad a sus docentes.

\section{Bibliografía}


Pineda, P. (2013). Formación integral para el manejo de entornos virtuales de aprendizaje por los docentes de la Faces UC. En: Eduweb, Revista de Tecnología y Comunicación en Educación. Vol. 7. No. 2. Universidad de Carabobo.

Prensky, M. (2001). Digital Natives, Digitals Inmigrants. En: On the Horizon. MCB University Press. Vol 9, No. 5.

Zumba, J. y Merchán, J. (2015). Disposición de los docentes para la incorporación de las Tecnologías de Información y Comunicación (TIC) como apoyo a los procesos de enseñanza y aprendizaje en la Universidad de Guayaquil En: Eduweb, Revista de Tecnología y Comunicación en Educación. Vol. 10. No. 2. Universidad de Carabobo.

Atillo.com. (2015). Universidades con carreras a distancia en Ecuador. Recuperado de: http://www.altillo.com/universidades/universidades_ecuol.asp

Cabero Almenara, J. (2008). La investigación en la educación a distancia en los nuevos entornos de comunicación telemáticos. Sociotam. Vol XVIII, N.2:13-34.

Castells, M. (2002). La dimensión cultural de Internet. Universitat Oberta de Catalunya. Recuperado de: http://www.uoc.edu/culturaxxi/esp/articles/castells0502/castells0502. html

Constitución de la República del Ecuador. (2008). Publicado en Registro Oficial \# 449, de fecha 20 de octubre de 2010. Quito.

Correa, C. (2013). Historia y tendencias de la educación a distancia en el Ecuador. En: Morocho, M y Rama, C. (Coord) La educación a distancia y virtual en Ecuador una nueva realidad universitaria. (156-172).Universidad Técnica Particular de Loja. Ecuador.

García Aretio, L. (1987). Hacia una definición de Educación a Distancia. Boletín informativo de la Asociación Iberoamericana de Educación Superior a distancia. Abril. Año 4, N ${ }^{\circ}$ $18,4 \mathrm{pp}$.

García Aretio, L. (2001). Bases conceptuales. Educación A Distancia. De la Teoría a la Práctica. Ariel Educación, Madrid, España. pp. 30-41.

Ley Orgánica de Educación Superior. (2010). Publicado en Registro Oficial No. 298, de fecha 12 de Octubre del 2010. Quito.

Rubio M., Morocho, M. y Ramírez, I. (2011). Leyes, Normas y Reglamentos que regulan la Educación Superior a Distancia y en Línea en Ecuador. En: Camacho, A. (Coord) Leyes, Normas y Reglamentos que regulan la Educación Superior a Distancia y en Línea en América Latina y el Caribe. (115-142). Universidad Técnica Particular de Loja. Ecuador.

Universidad de Guayaquil. (2015). Nosotros. Recuperado de: http://www.ug.edu.ec/nosotros/ 
Nuñez, T. F. (2011). Entornos virtuales de enseñanza-aprendizaje (EVEA): Formación profesional. Edutec-e, Revista Electrónica de Tecnología Educativa, 37. Recuperado de: http://edutec.rediris.es/revelec2/revelec37/entornos_virtuales_ensenanza_formacion_ profesional.html

García Martínez, T., García Mauricio J. C. (2013). Aplicación de cuestionarios online sobre el rendimiento académico Revista de Informática Educativa y Medios Audiovisuales, Vol. 10(17):1-7. Recuperado de:http://laboratorios.fi.uba.ar/lie/Revista/Articulos/101017/ A1mar2013.pdf 